\title{
Left Atrial Stiffness, a Marker of Atrial Cardiomyopathy, and Atrial Fibrillation - Relationships and Predictors for Procedure Success after Catheter Ablation
}

\author{
Tan Chen $\mathrm{Wu}^{(0)}$ \\ Instituto do Coração - Faculdade de Medicina da Universidade de São Paulo, São Paulo, SP - Brazil \\ Short Editorial related to the article: Left Atrial Stiffness: A Predictor of Atrial Fibrillation Recurrence after Radiofrequency Catheter Ablation - \\ A Systematic Review and Meta-Analysis
}

Over the past years, catheter ablation (CA) for atrial fibrillation (AF) has established itself as a well-recognized strategy in the management of patients with $\mathrm{AF}$ and an important option for rhythm control. Although CA is more effective than antiarrhythmic drug therapy, AF recurrences are common during the follow-up. ${ }^{1}$

Late recurrence, during the first 9 months after the blanking period, occurs in $25 \%-40 \%$ of cases and is predominantly linked to the recovery of electrical conduction between the pulmonary veins (PVs) and the left atrium (LA), irrespective of the type of AF. The incidence of very late recurrence (after more than 12 months postablation) has been shown to be higher than previously expected, with an annual recurrence rate estimated at $7.6 \% .^{2}$ Bunch et al. ${ }^{3}$ reported $\mathrm{AF}$ recurrence rates ranging from $52 \%$ ( $\leq 50$ years + paroxysmal $\mathrm{AF}$ ) to $75 \%$ $\left(>80\right.$ years + paroxysmal AF). ${ }^{3}$ In a series of 509 consecutive patients undergoing paroxysmal AF ablation by Teunissen et al., after a single procedure, antiarrhythmic drugs free success rate was $41.3 \%{ }^{4}$. The predominant mechanism of very late recurrence includes, in addition to the PV connection, the development of non-PV triggers, and development and maturation of substrate. The predictors appears to be the nonparoxysmal form of AF at baseline, organic heart disease, advanced age, and obesity.

$\mathrm{AF}$ is often associated with atrial structural remodeling and causes LA fibrosis/scarring and dilatation. Substrate progression is a multifactorial and time-dependent response of cardiac myocytes to varying "stressors", including electrical, mechanical, and metabolic stressors. Some components of the LA changes are reversible (adaptive), whereas others are permanent (maladaptive). Most risk factors affect AF by causing structural remodeling. Progression of atrial damage due to underlying heart disease is a major factor. Recent studies suggest that AF recurrence can be prevented by effectively managing risk factors such as sleep apnea, obesity, high blood pressure,

\section{Keywords}

Atrial Fibrillation; Catheter Ablation; Atrial Function, Left; Atrial Remodeling; Recurrence; Treatment Outcome.

Mailing Address: Tan Chen Wu •

Unidade de Arritmia do Instituto do Coração da Faculdade de Medicina da Universidade de São Paulo - Av. Dr. Eneas Carvalho de Aguiar, 44. Postal

Code 05403-000, São Paulo, SP - Brazil

E-mail: tanchen.cardio@gmail.com

DOI: 10.5935/abc.20190087 hyperglycemia, and dyslipidemia, presumably by curtailing further damage and/or reversing existing abnormalities. Conversely, AF itself can cause progression of the substrate. In addition to complexion-channel remodeling that accelerates repolarization and alters conduction properties, rapid activation of atrial cardiomyocytes causes profibrotic changes in fibroblast function and promotes atria fibrosis.

Increased LA scar is associated with increases LA stiffness, which reflects a deteriorated reservoir function. Therefore, LA stiffness could be associated with LA histological changes and predicts sinus rhythm maintenance after treatment in AF patients. ${ }^{5}$ Timely intervention for patients with these conditions may interrupt and perhaps reverse LA remodeling, with a consequent reduction in LA size and improved function.

The scar tissue formation after CA may also adversely impact the diastolic properties of the LA, especially after multiple ablation procedures, worsening the diastolic function or LA compliance. Stiff LA syndrome has been recognized as pulmonary hypertension and dyspnea that develops after CA, a potential complication of the procedure with a low prevalence. ${ }^{6,7}$

Thus, evaluation of the LA as cardiovascular biomarker, especially in $\mathrm{AF}$, has become increasingly important. ${ }^{8,9}$ LA remodeling is monitored in clinical practice using various noninvasive imaging modalities, but it has not been yet incorporated into clinical decision making. In this published issue, Correia et al., ${ }^{10}$ investigated, through a systematic review and meta-analysis, if LA stiffness could be a predictor of $\mathrm{AF}$ recurrence after $\mathrm{CA}$, and to discuss its clinical use. ${ }^{10}$ Only 4 prospective observational studies were included in the systematic review and 3 of them in the meta-analysis, with different methods, and most of all used LA pressure measured invasively during CA to estimate LA stiffness. They found that LA stiffness was a strong independent predictor of $\mathrm{AF}$ recurrence after $\mathrm{CA}(\mathrm{HR}=3.55,95 \% \mathrm{Cl} 1.75-4.73$, $\mathrm{p}=0.0002$ ), and concluded that a non-invasive assessment of LA stiffness prior to CA can be used as a potential screening factor to select or to closely follow patients with higher risks of AF recurrence and development of the stiff LA syndrome. The small number of studies, with heterogeneity and a short mean follow-up in 3 studies were limitations in this meta-analysis.

These findings add to our knowledge by clarifying the association between atrial remodeling and outcomes after AF ablation. Current guidelines recommendation is to perform $\mathrm{CA}$ as second-line treatment after failure or intolerance to at least one antiarrhythmic drug. As first-line treatment, the 
indication recommendations are weaker and only limited to patients with paroxysmal AF. These recommendations usually lead physicians to treat patients with CA after a longer history period of clinical AF. The development of tools and methods to determine markers of atrial cardiomyopathy may allow to avoid the mismatch of the best time for CA, in

\section{References}

1. Ha AC, Wijeysundera HC, Birnie DH, Verma A. Real-world outcomes, complications, and cost of catheter-based ablation for atrial fibrillation: an update. Curr Opin Cardiol. 2017;32(1):47-52

2. Calkins H, Hindricks G, Cappato R, Kim YH, Saad EB, Aguinaga, et al. 2017 HRS/EHRA/ECAS/APHRS/SOLAECE expert consensus statement on catheter and surgical ablation of atrial fibrillation. Europace. 2018;20(1):e1-e160.

3. Bunch TJ, May HT, Bair TL, Jacobs V, Crandall BG, Cutler M, et al. The impact of age on 5-year outcomes after atrial fibrillation catheter ablation. J Cardiovasc Electrophysiol. 2016 Feb;27:141-6.

4. Teunissen C, Kassenberg W, van der Heijden JF, Hassink RJ, Vincent JHM, Nidces PA. Five-year efficacy of pulmonary vein antrum isolation as a primary ablation strategy for atrial fibrillation: a single-centre cohort study. Europace. 2016;18(9):1335-42.

5. Thomas L, Abhayaratna WP. Left atrial reverse remodeling. mechanisms, evaluation, and clinical significance. JACC Cardiovascular Imaging. 2017;10(1):65-77. accordance with more substrate and patient-oriented process of diagnosis and therapy of AF. Certainly, further studies will be required to support identification by noninvasive cardiac imaging of patients for whom CA should be considered early before there is significant LA functional remodeling with associated fibrosis.
6. Leite-Moreira AF, Oliveira SM, Marino P. Left atrial stiffness and its implications for cardiac function. Future Cardiol. 2007; 3(2):175-183

7. Gibson DN, Di Biase L, Mohanti P, Patel JD, Bai R, Sanchez J, et al. Stiff left atrial syndrome after catheter ablation for atrial fibrillation: clinical characterization, prevalence, and predictors. Heart Rhythm. 2011;8(9):1364-71

8. Njoku A, Kannabhiran M, Arora R, Reddy P, Gopinathannair R, Lakkireddy $D$, et al. Left atrial volume predicts atrial fibrillation recurrence after radiofrequency ablation: a meta-analysis. Europace. 2018;20(1):33-42.

9. Ma XX, Boldt LH, Zhang YL, Zhu MR, Hu B, Parwani A, et al. Clinical relevance of left atrial strain to predict recurrence of atrial fibrillation after catheter ablation: a meta-analysis. Echocardiography. 2016;33(5):724-33.

10. Correia ETO, Barbetta LMS, Silva OMP, Mesquita ET. Left atrial stiffness: a predictor of atrial fibrillation recurrence after radiofrequency catheter ablation - a systematic review and meta-analysis. Arq Bras Cardiol. 2019; 112(5):501-508 